\title{
Distances of the bulge globular clusters Terzan 5, Liller 1, UKS 1, and Terzan 4 based on HST NICMOS photometry
}

\author{
S. Ortolani ${ }^{1}$, B. Barbuy ${ }^{2}$, E. Bica ${ }^{3}$, M. Zoccali ${ }^{4}$, and A. Renzini ${ }^{5, \star}$
}

1 Università di Padova, Dipartimento di Astronomia, Vicolo dell'Osservatorio 2, 35122 Padova, Italy e-mail: sergio.ortolani@unipd.it

2 Universidade de São Paulo, IAG, Rua do Matão 1226, Cidade Universitária, São Paulo 05508-900, Brazil e-mail: barbuy@astro.iag.usp.br

3 Universidade Federal do Rio Grande do Sul, Departamento de Astronomia, CP 15051, Porto Alegre 91501-970, Brazil e-mail: bica@if.ufrgs.br

4 Universidad Catolica de Chile, Department of Astronomy \& Astrophysics, Casilla 306, Santiago 22, Chile e-mail: mzoccali@astro.puc.cl

5 Osservatorio Astronomico di Padova, Vicolo dell'Osservatorio 5, I-35122 Padova, Italy e-mail: alvio.renzini@oapd.inaf.it

Received 24 October 2006 / Accepted 10 May 2007

ABSTRACT

\begin{abstract}
Context. A large number of pulsars and X-rays sources are detected in globular clusters. To understand the structure and content of these clusters, accurate distances are required.

Aims. We derive the distances of Terzan 5, Liller 1 and UKS 1 using as a reference a recent distance determination of NGC 6528, based on HST/NICMOS and NTT/SOFI infrared photometry. The distance of the metal-poor cluster Terzan 4 was derived from a comparison with M92 in NICMOS bands.

Methods. Distances of the metal-rich clusters are obtained by comparison of the Horizontal Branch (HB) level of the clusters, relative to the reddening line passing through the HB of NGC 6528. We use methods based on NICMOS bands and transformations to $J$ and $H$ magnitudes with different assumptions.

Results. Liller 1 and Terzan 4 are found to be at the central bulge distance, UKS 1 is beyond the Galactic center, while Terzan 5 is closer to the Sun than the other four clusters.

Conclusions. The distance of Terzan 5 is of paramount importance, given the impact of its population of 21 pulsars, which is related to the high cluster density. The distance of Terzan 5 is found to be $d_{\odot}=5.5 \pm 0.9 \mathrm{kpc}$ from the Sun, thus closer to us than values given in studies of pulsars in Terzan 5. As a consequence, the higher cluster density is even more favourable for formation of the millisecond pulsars recently detected in this cluster.
\end{abstract}

Key words. Galaxy: bulge - Galaxy: globular clusters: individual: NGC 6528 - Galaxy: globular clusters: individual: Terzan 5 Galaxy: globular clusters: individual: Liller 1 - Galaxy: globular clusters: individual: Terzan 4 -

Galaxy: globular clusters: individual: UKS 1

\section{Introduction}

Globular cluster distances are fundamental to studies of the Galaxy structure and stellar population components (e.g. Bica et al. 2006). Distances of clusters near the Galactic center are particularly interesting, since several of them host a large number of X-ray sources and pulsars (Cackett et al. 2006; Ransom et al. 2005; Lorimer 2005). Accurate distances are crucial to estimate cluster stellar densities, which in turn affect stellar interaction rates in globular clusters, where dense environments favour formation of binaries. Verbunt \& Hut (1987; see also Hut et al. 1992) reported that $20 \%$ of low-mass X-ray binaries are found in globular clusters. They also pointed out that Terzan 5 and Liller 1 might be the densest clusters in the Galaxy. Ransom et al. (2005) and Lorimer (2005) reported 21 pulsars in Terzan 5. Camilo \& Rasio (2005) reported that Terzan 5 and 47 Tucanae together have 45 millisec pulsars, as a probable result of the "recycling" model (where an accreting neutron star is spun up

* Based on observations collected with the NASA/ESA Hubble Space Telescope obtained at the Space Telescope Science Institute, which is operated by the Association of Universities for Research in Astronomy, Inc., under NASA contract NAS 5-16555. and becomes a fast radio pulsar). This model connects low mass $\mathrm{X}$-ray binaries (LMXB) to millisecond pulsars (MSP).

The metal-rich globular cluster NGC 6528 is a template object of the bulge population (Bica 1988; Ortolani et al. 1992; Ortolani et al. 1995), and is used here as a reference. The distance of NGC 6528 was derived by Momany et al. (2003) from a combination of proper motion cleaned optical and infrared Colour-Magnitude Diagrams (CMDs), obtained from images observed with HST-WFPC2, and the ESO NTT-SOFI infrared camera. The resulting CMDs were compared to fiducial lines of 47 Tucanae and NGC 6553 and to Padova isochrones. A distance modulus of $(m-M)_{\circ}=14.44 \pm 0.06$ and a distance from the Sun $d_{\odot}=7.7 \pm 0.2 \mathrm{kpc}$ were obtained, where the errors were derived from a comparison between empirical and a theoretical calibration of the HB. The distance errors in the infrared are small relative to the optical, because of the minimized reddening dependence. Momany et al.'s cleaned CMDs show unprecedented accuracy at the HB level of $K(\mathrm{HB})=13.20 \pm 0.05$. A re-check taking into account calibration uncertainties, indicates that the distance modulus error should be of the order of \pm 0.10 . Note that the distance of the Galactic center considered previously to be at $8 \mathrm{kpc}$ (Reid 1993) has been recently revised to be closer to 
the Sun at $7.62 \pm 0.32 \mathrm{kpc}$ (Eisenhauer et al. 2005), $7.5 \pm 0.1 \mathrm{kpc}$ (Nishiyama et al. 2006) and 7.2 \pm 0.3 (Bica et al. 2006).

Ortolani et al. (2001) studied HST-NICMOS ColourMagnitude Diagrams of the reddened bulge globular clusters NGC 6528, Terzan 5, Liller 1, Terzan 4 and UKS 1, to derive their ages. The results confirmed that these bulge clusters are old, with ages comparable with that of 47 Tucanae, as previously found by Ortolani et al. (1995). In the present work, we concentrate efforts to derive distances for this sample, taking NGC 6528 as a reference.

From the same NICMOS data, Cohn et al. (2002) derived a distance of Terzan 5 from the Sun of $d_{\odot}=8.7 \mathrm{kpc}$. From optical NTT-SUSI photometry, Ortolani et al. (1996a) derived $d_{\odot}=5.6 \mathrm{kpc}$. In the present paper, we readdress the Terzan 5 distance issue, given its impact on the cluster density implying in binary and pulsar formation. Matsunaga et al. (2005) searched for $\mathrm{SiO}$ masers in globular clusters and a very luminous one was found in the central part of Terzan 5, proposed to result from the merging of two AGB stars, likely to occur at this extreme stellar density. Also, Cohn et al. (2002) reported an RR Lyrae in Terzan 5, the first detected at such high metallicities, and it was interpreted as due to interaction effects. Finally, Heinke et al. (2006) detected 50 faint X-ray sources in Terzan 5, based on observations with the Chandra satellite.

Distances based on optical CMDs were derived for the bulge globular clusters Terzan 4 (Ortolani et al. 1997a), Terzan 5 (Ortolani et al. 1996a), Liller 1 (Ortolani et al. 1996b) and UKS 1 (Ortolani et al. 1997b). The purpose of this paper is to derive more accurate distances for these clusters making use of NICMOS data, as well as to better understand the large number of pulsars in Terzan 5. Distances of central clusters are also important to establish a locus of survival of old star clusters in the central regions of the Galaxy.

The observations and reductions are briefly reported in Sect. 2. In Sect. 3 we discuss cluster metallicities, based on the Red Giant Branch (RGB) slope in the infrared. In Sect. 4 calibrations of NICMOS $m_{110}$ and $m_{160}$ photometry are discussed. In Sect. 5, distances derived in the present work are presented. In Sect. 6 implications for the pulsar rich cluster Terzan 5 are developed. Concluding remarks are given in Sect. 7. Details on calibrations of NICMOS photometry are given in Appendix A.

\section{Observations and reductions}

Ortolani et al. (2001) observed the sample globular clusters with NICMOS on board HST, through the F110W and F160W filters, using NIC1 and NIC2 cameras. The present analysis is based

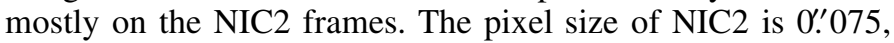
giving a field of view of $19.2 \times 19.2$ for each frame. The description of data, reduction, and a Log of the observations are detailed in Ortolani et al. (2001). The typical FWHM of the stars was $0 . ' 11$. The zero point PHOTZPT was applied to transform instrumental magnitudes into the standard HST magnitudes $m_{110}$ and $m_{160}$.

All the CMDs reach well below the HB. The CMDs of NGC 6528 and Terzan 4 reach more than 3 mag below the turnoff in both the NIC1 and NIC2-offset pointings. The deepest CMD for Terzan 5 reaches about 1 mag. below the turnoff.

\section{Metallicities of the sample clusters}

Metallicities of globular clusters can be estimated from the RGB slope as previously applied to optical and infrared CMDs (Ortolani et al. 1990, 1991; Tiede et al. 1997).
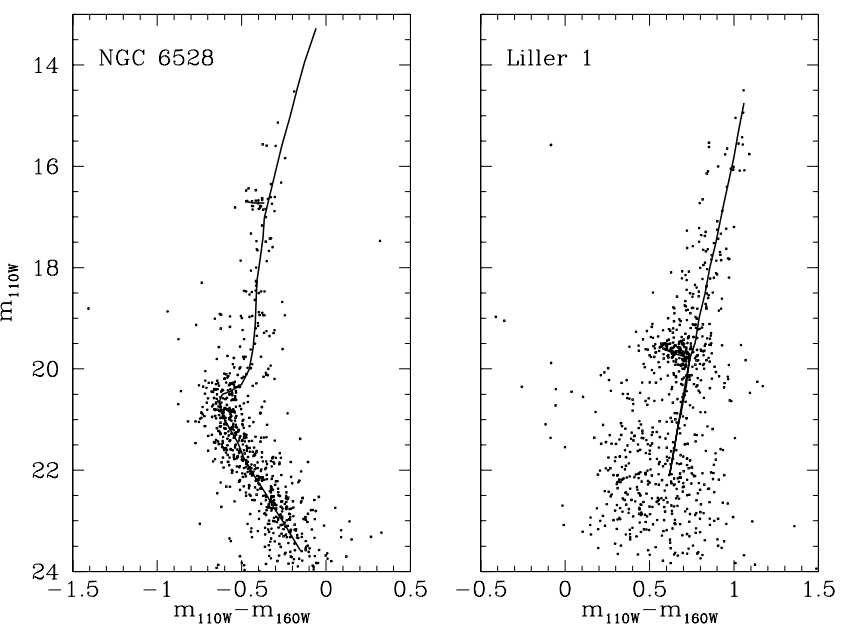

Fig. 1. Colour-magnitude diagrams and mean loci in $m_{110}$ vs. $m_{110}-$ $m_{160}$ for NGC 6528 and Liller 1.
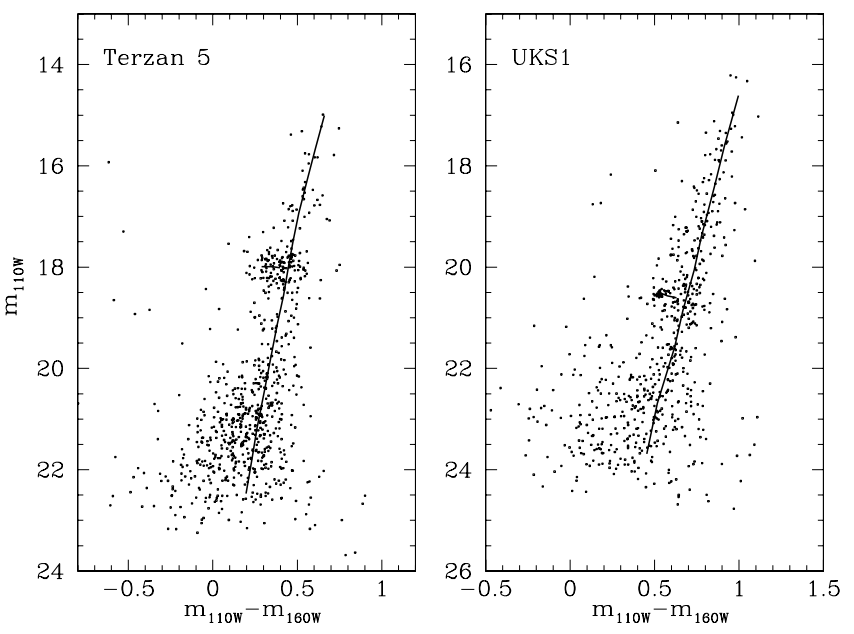

Fig. 2. Colour-magnitude diagrams and mean loci in $m_{110}$ vs. $m_{110}-$ $m_{160}$ for Terzan 5 and UKS 1.

In Figs. 1 and 2 we show the individual CMDs of the four metal-rich sample clusters, and corresponding mean loci, plotted by eye. We estimate an uncertainty of \pm 0.1 in both $m_{110}$ and $m_{110}-m_{160}$. In Fig. 3 we also show the metal-poor cluster Terzan 4 (Sect. 4.1).

In Fig. 4 are plotted the mean loci of the sample clusters, by matching their HB level, and subgiant branch (SGB) at the HB colour, or the turn-off in the case of Terzan 4. It appears that NGC 6528 is the most metal-rich cluster of the sample, closely followed by Terzan 5, and next by Liller 1 and UKS 1 , in this order. The RGB of Terzan 4 is steeper due to its low metallicity. The RGB slope measured in the CMD of NGC 6528 is $\Delta m_{110} / \Delta\left(m_{110}-m_{160}\right)=11.0$, while for UKS 1 it is $\Delta m_{110} / \Delta\left(m_{110}-m_{160}\right)=15.0 \pm 1.0$, the latter value being the most uncertain one.

Recent high resolution spectroscopic determinations confirm this ranking for NGC 6528 (Zoccali et al. 2004), Terzan 5 and Liller 1 (Origlia et al. 2002; Origlia \& Rich 2004), and UKS 1 (Origlia et al. 2005).

Reddening and metallicity values for the sample clusters revised by Barbuy et al. (1998) and the compilation by Harris (1996), as given at http://www.physics.mcmaster.ca/ Globular.html, as well as recent spectroscopic determinations are shown in Table 1. 


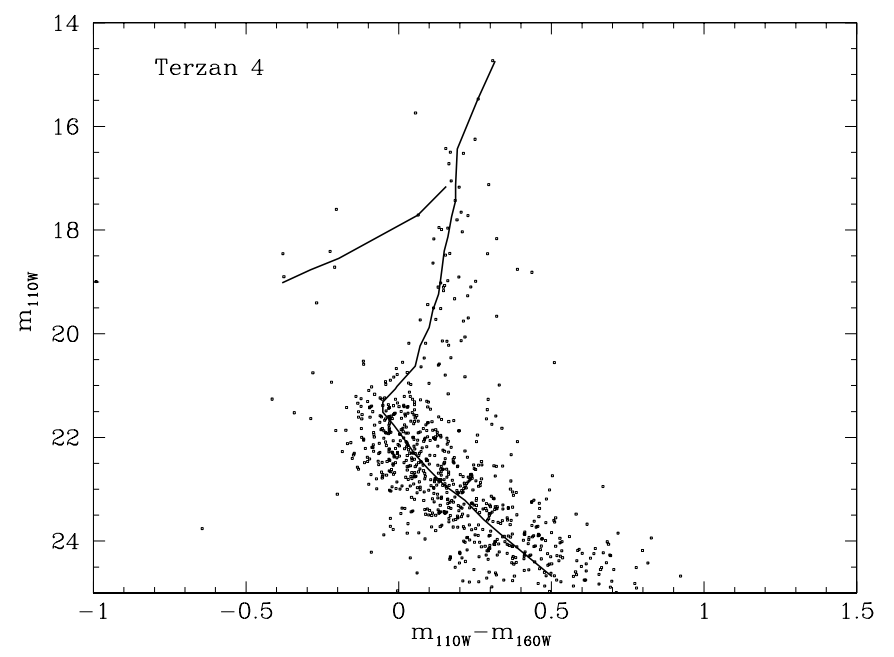

Fig. 3. Colour-magnitude diagram of Terzan 4, and mean loci of M92 overplotted, in $m_{110}$ vs. $m_{110}-m_{160}$.

\section{Distance from NICMOS instrumental magnitudes}

For the derivation of distance, two methods are possible, one using comparisons with template clusters in the instrumental NICMOS bands, and the second by transforming them into $J$ and $H$.

Schlegel et al. (1998)'s reddening law (1998) was employed to obtain the infrared extinction slope $A_{110} /\left(A_{110}-A_{160}\right)$ in the HST/NICMOS bands: $A_{110} / E(B-V)=1.186, A_{160} / E(B-V)=$ 0.634 , and $A_{110} /\left(A_{110}-A_{160}\right)=2.148$. As a check, we also calculated the slope using the Lee et al. (2001) NICMOS extinction law based on synthetic spectra, obtaining a similar value $A_{110} /\left(m_{110}-m_{160}\right)=2.03$.

Figure 5 shows the observed mean loci for the 5 clusters, in NICMOS $m_{110}$ vs. $m_{110}-m_{160}$. The reddening line $A_{110} / E\left(m_{110}-\right.$ $\left.m_{160}\right)=2.148$ passing through the HB of NGC 6528 is also shown in Fig. 5. Were all the clusters at the same distance, then the HB of all of them would be on the same line. Therefore, the $m_{110}$ difference between each cluster HB and the reddening line represents the distance modulus difference between NGC 6528 and the cluster, assuming that they share the same metallicity (or same intrinsic SGB colour and HB absolute value). The advantage of this method is that reddening from NICMOS colours is not needed.

Figure 5 shows that Liller 1 and NGC 6528 differ significantly in reddening. Terzan 5 is located above the reddening line, thus at a shorter distance, whereas UKS 1 is below the line, and therefore farther than NGC 6528. The distances derived are given in Col. 5 of Table 2.

\subsection{Distance of the metal-poor cluster Terzan 4}

Given the difference in Horizontal Branch morphology between metal-poor and metal-rich clusters, the method described in Sect. 4 cannot be applied to Terzan 4 . The distance of Terzan 4 was derived by comparing its CMD to the mean locus of M 92 (Lee et al. 2001) observed in the same NICMOS bands (Fig. 3). Lee et al.'s reddening law in the NICMOS bands was adopted. The best fit gives $\Delta\left(m_{110}-m_{160}\right)(\mathrm{Tz} 4-\mathrm{M} 92)=0.73 \pm 0.02$ and $\Delta\left(m_{110}\right)(\mathrm{Tz} 4-\mathrm{M} 92)=1.6 \pm 0.1$. Adopting $E(B-V)=$ $1.87 E\left(m_{110}-m_{160}\right)=1.38$ and $A_{110} / E(B-V)=1.16$ (Lee et al. 2001), we get $A_{110}=1.38 \times 1.16=1.60$ and $\Delta(m-$ $M)_{\circ}(\mathrm{Tz} 4-\mathrm{M} 92)=0.0$. Finally $A_{\mathrm{V}}=1.38 \times 3.3=4.5 \mathrm{mag}$.

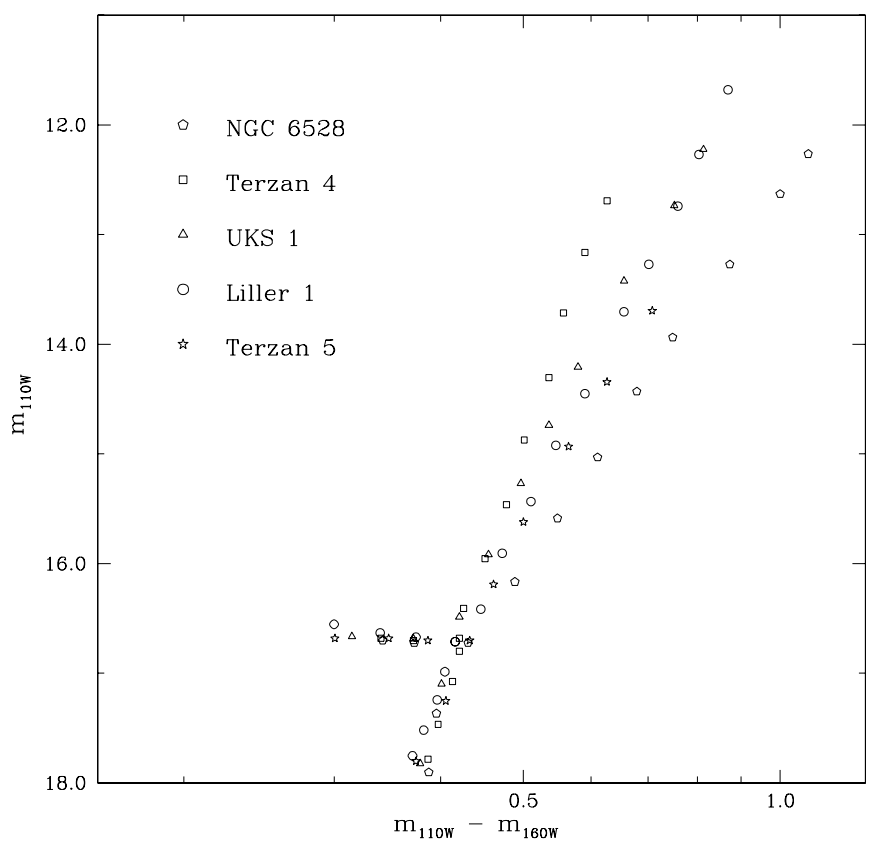

Fig. 4. Mean loci in $m_{110}$ vs. $m_{110}-m_{160}$ for the five sample clusters, by matching relative to NGC 6528 their HBs and SGB @ HB, or the turnoff in the case of Terzan 4. NGC 6528: black circles Terzan 5: open triangles; UKS 1: black triangles; Liller 1: open pentagon; Terzan 4: black square.

This means that Terzan 4 is at the same distance as M92, which is $8 \mathrm{kpc}$ according to Harris (1996). Therefore the distance of Terzan 4 is $R_{\odot}=8.0 \pm 0.3 \mathrm{kpc}$. This is very close to the Galactic center. Its extinction is rather below the 7 mag we derived in the optical, but the distance is compatible with our previous estimations of $8.3 \pm 0.7 \mathrm{kpc}$ (Ortolani et al. 1997a) and $7.3 \mathrm{kpc}$ (Barbuy et al. 1998). The present determination gives the most reliable distance and reddening values for Terzan 4.

\subsection{Errors}

Distance errors in the method described above are dominated by uncertainties on:

(a) The measurements of SGB colours and luminosities at the level of the HB. Differential reddening across the images is the main source of errors, and such effects are common to all calibrations. Differential reddening together with some field contamination of the lower Galactic latitude clusters (such as Liller 1) are the main causes of scattering in the CMDs. The intrinsic photometric errors are negligible because of the very high signal to noise ratio at the $\mathrm{HB}$ level, given that the photometry reaches below the turnoff. In the CMDs they are of the order of 0.07 in colour and 0.15 in magnitude. These values were obtained by measuring the HB in different parts of the images and/or comparing NIC1 and NIC2 cameras. However, since the CMD scatter is mainly in the direction of the reddening line, the effect propagated on the distance is reduced to about $0.1 \mathrm{mag}$. A metallicity uncertainty of about 0.2 dex would imply the same error (0.1 mag).

(b) The slope of the reddening law. The formal error on the distances introduced by the uncertainty on the slope of the reddening line (Schlegel et al. 1998; Fitzpatrick 1999) is considerably lower, of the order of $0.02 \mathrm{mag}$ in the full colour range. Second order effects may arise due to the wide 
Table 1. Literature reddening and metallicity (Barbuy et al. 1998; Harris 1996 and high resolution spectroscopy by 1 Origlia \& Rich 2004; 2 Carretta et al. 2001; 3 Zoccali et al. 2004; 4 Origlia et al. 2005; 5 Origlia et al. 2002).

\begin{tabular}{|c|c|c|c|c|c|c|c|c|}
\hline \multirow[b]{2}{*}{ Cluster } & \multirow[b]{2}{*}{$l$} & \multirow[b]{2}{*}{$b$} & \multicolumn{2}{|c|}{ Barbuy et al. 1998} & \multicolumn{2}{|c|}{ 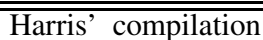 } & \multirow[b]{2}{*}[\mathrm{Fe}/\mathrm{H}]{ spec } & \multirow[b]{2}{*}{ ref. } \\
\hline & & & $E(B-V)$ & {$[\mathrm{Fe} / \mathrm{H}]$} & $E(B-V)$ & {$[\mathrm{Fe} / \mathrm{H}]$} & & \\
\hline NGC 6528 & 1.14 & $-4^{\circ} .18$ & 0.52 & -0.2 & 0.56 & -0.17 & -0.1 & 2,3 \\
\hline Terzan 5 & $3: 81$ & 1.67 & 2.39 & 0.0 & 2.37 & -0.28 & -0.21 & 1 \\
\hline Liller 1 & $354^{\circ} .81$ & -0.16 & 3.0 & +0.2 & 3.00 & +0.22 & -0.3 & 5 \\
\hline UKS 1 & 5.13 & 0.76 & 3.1 & -0.5 & 3.09 & -0.5 & -0.78 & 4 \\
\hline Terzan 4 & $356^{\circ} .02$ & 1.31 & 2.31 & -2.0 : & 2.35 & -1.60 & -1.6 & 1 \\
\hline
\end{tabular}

Table 2. Horizontal branch magnitudes, colours and distances for different photometric calibrations. The $m_{110}$ and $J$ magnitudes refer to the HB, colours refer to the SGB at the HB level. $\Delta m_{110}$ and $\Delta J$ are the distance moduli differences relative to the reference cluster NGC 6528 . Assuming for UKS 1 and Liller 1 a metallicity of ${ }^{a}$ the same as NGC 6528 of $[\mathrm{Fe} / \mathrm{H}]=-0.1 ;{ }^{b}$ more probable value of $[\mathrm{Fe} / \mathrm{H}]=-0.6$.

\begin{tabular}{lccccccccccccc}
\hline \hline & \multicolumn{3}{c}{ NICMOS instrumental } & \multicolumn{4}{c}{ Stephens et al. calibration } & \multicolumn{4}{c}{ Cohn et al. calibration } \\
\cline { 2 - 11 } Cluster & $m_{110}-m_{160}$ & $m_{110}$ & $\Delta m_{110}$ & $d(\mathrm{kpc})$ & $J(\mathrm{HB})$ & $J-H(\mathrm{HB})$ & $\Delta J$ & $E(B-V)$ & $d(\mathrm{kpc})$ & $J(\mathrm{HB})$ & $\Delta J$ & $E(B-V)$ & $d(\mathrm{kpc})$ \\
\hline NGC 6528 & -0.36 & 16.71 & - & 7.7 & 14.2 & 0.58 & - & 0.46 & 7.7 & 14.11 & - & 0.46 & 7.7 \\
Terzan 5 & 0.44 & 17.99 & -0.44 & 6.3 & 15.22 & 1.19 & -1.10 & 2.82 & 4.6 & 15.09 & -0.70 & 2.33 & 5.6 \\
Liller 1 $^{a}$ & 0.75 & 16.66 & 0.57 & 10.0 & 16.79 & 1.42 & -0.36 & 3.74 & 6.5 & 16.79 & 0.36 & 3.04 & 9.1 \\
Liller 1 $^{b}$ & & & & 8.9 & & & & & 5.8 & & & 8.1 \\
UKS 1 $^{a}$ & 0.70 & 20.56 & 1.57 & 15.9 & 17.69 & 1.39 & 0.68 & 3.59 & 10.6 & 17.69 & 1.34 & 2.94 & 14.3 \\
UKS 1 $^{b}$ & & & & 14.2 & & & & & 9.3 & & & 12.9 \\
\hline
\end{tabular}

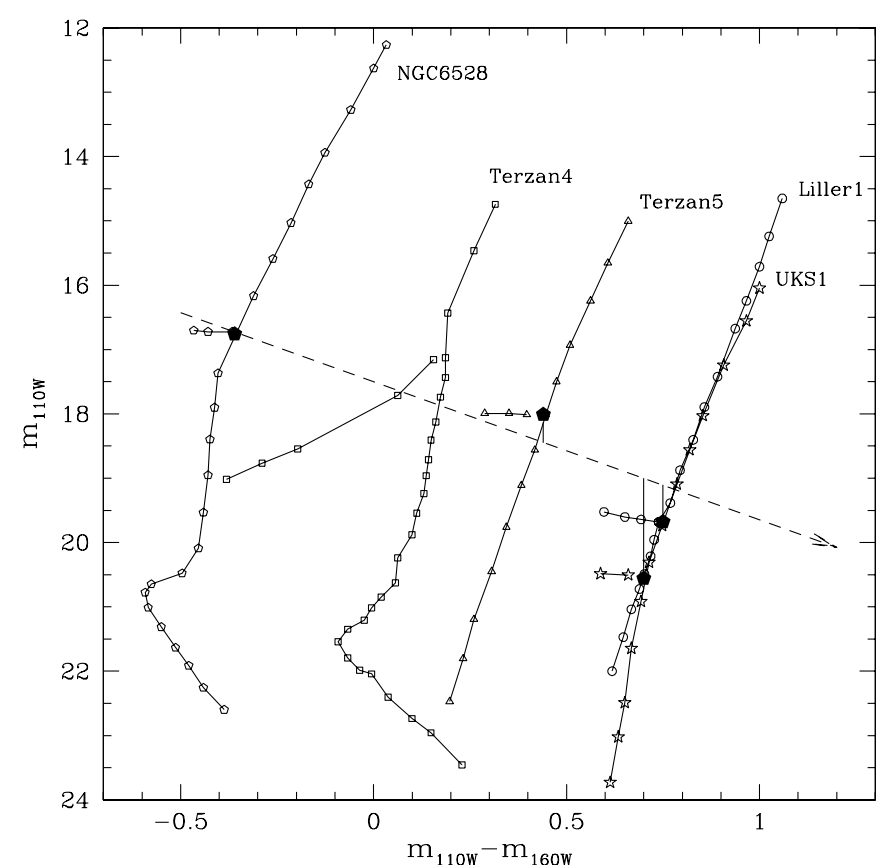

Fig. 5. Mean loci in $m_{110}$ vs. $m_{110}-m_{160}$ for the five sample clusters, in ST magnitudes. The solid line is the reddening line with a slope $A_{110} / E\left(m_{110-160}\right)=2.15$, passing through NGC 6528. NGC 6528: open circles; Terzan 4: open squares; Terzan 5: open triangles; Liller 1: open circles; UKS 1: open stars.

passband of the F110W and F160W filters (the first is about twice that of the standard $J$, and the second $35 \%$ wider than the $H$ band). Possible non linear effects due to the distorted passbands may be an important uncertainty of this calibration and cannot be easily quantified. While the second order reddening effects are well known in the optical photometry, in the infrared the effects on the standard $K$ band have been only recently inferred (Kim et al. 2005).

\section{Reddening and distance from NICMOS calibrated data}

$J$ and $H$ calibrated magnitudes and colours have been obtained by adopting linear transformation equations by Stephens et al. (2000) and Cohn et al. (2002). Both authors used the NICMOS Data Handbook standard stars, whereas Stephens et al. added Baade's Window giants observed both with NICMOS and from ground in $J H K$. More details on the calibrations are given in the Appendix.

In Fig. 6 we show the $J$ level of the HB vs. $J-H$ of the SGB at the HB level (SGB@HB) for the 4 metal-rich sample clusters. In Figs. 5 and 6, the distance determination is independent of reddening values and zero point calibration. A source of uncertainty is the choice of the reddening law, but differences between them in the infrared are small (Schlegel et al. 1998; Fitzpatrick 1999). Moreover, there is a general agreement in the literature that, in the infrared, the reddening slope is constant and independent of the dust characteristics.

Transformations of $E(J-H)$ into $E(B-V)$ are reported in the Appendix. Assuming for NGC 6528 the reddening $E(B-V)=$ 0.46 (Zoccali et al. 2004), we get for Terzan 5, Liller 1 and UKS 1 the $\Delta E(B-V)$ values. In order to obtain the total reddening, that of NGC 6528 itself has to be added, and the results are given in Table 2 (Cols. 9 and 13 for the two calibrations respectively). A good agreement is found with optical CMDs (Table 1) for Terzan 5. For Liller 1 and UKS 1, Cohn et al.'s calibration is more compatible with the literature.

In Table 2 are given distance determinations for the sample clusters, assuming for NGC 6528 the value $d_{\odot}=7.7 \mathrm{kpc}$ from Momany et al. (2003), and using the independent calibrations from instrumental to $J$ and $H$ bands by Stephens et al. (2000) and Cohn et al. (2002). Columns 8 and 12 of Table 2 give the distance modulus difference between each cluster and NGC 6528, measured along the reddening line (Figs. 5 and 6), for each calibration. This makes this procedure reddening independent. The differences in distances between the two calibrations arise from 


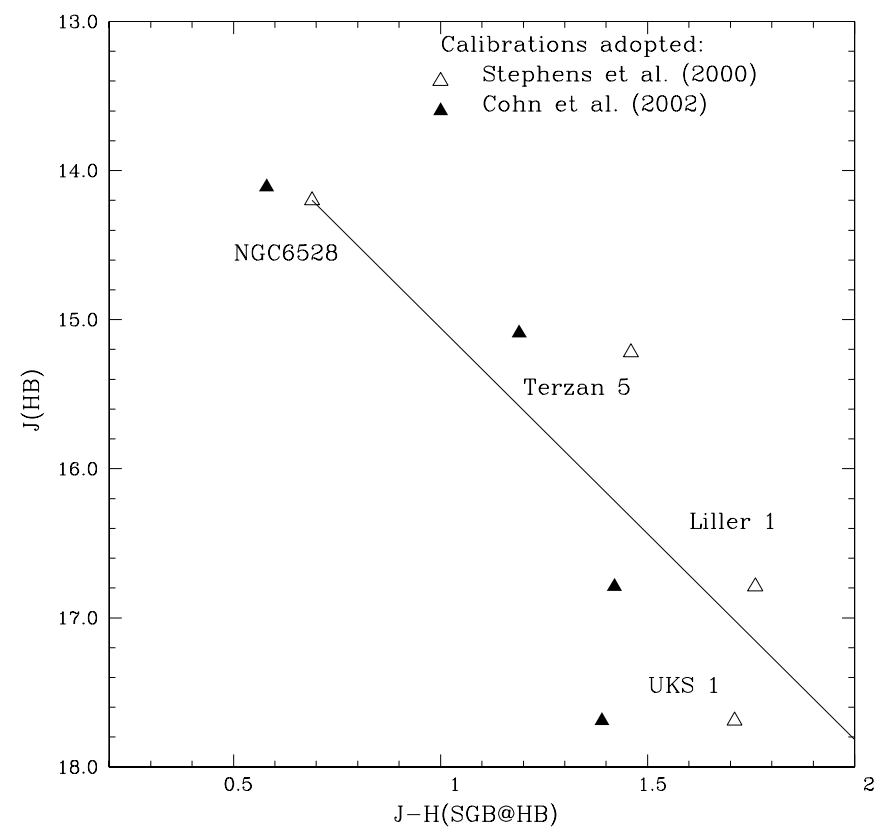

Fig. 6. $J$ level of the HB vs. $J-H$ of the SGB at the HB level for the four metal-rich clusters, derived with two different transformation equations. The solid line is the reddening line with a slope $A_{J} / E(J-H)=2.76$, passing through NGC 6528. Symbols: open triangles: transformation equations by Stephens et al. (2000); filled triangles: transformation equations by Cohn et al. (2002).

the different calibration coefficients, and not from photometric zero points.

In Table 2 we calculated the distances of UKS 1 and Liller 1 for two metallicity values: the same as that of NGC 6528 $([\mathrm{Fe} / \mathrm{H}]=-0.1)$, and a correction of $\Delta[\mathrm{Fe} / \mathrm{H}]=0.5$, making use of Padova isochrones (Girardi et al. 2000). The colour changes by $\Delta(J-H) \approx 0.1$, corresponding to a decrease of 0.25 magnitudes in the distance modulus.

Terzan 5 distance estimates of 4.6 and $5.6 \mathrm{kpc}$, put it closer than the Galactic center, whereas a mean of 6.5 and $9.1 \mathrm{kpc}$ for Liller 1 place the cluster near the Galactic center. UKS 1 estimates of 9.3 to $12.9 \mathrm{kpc}$ suggest that the cluster is beyond the Galactic center.

\section{Pulsars and density of Terzan 5}

Terzan 5 distance estimates range from 4.6 to $6.3 \mathrm{kpc}$ (Table 2), shorter than Cohn et al. (2002)'s value of $8.7 \mathrm{kpc}$. Cohn et al. derived this distance in two ways: (i) One using a comparison of HB level of NGC 6528 in the $H$ band, adopting the dereddened $H(\mathrm{HB})=13.1$ from Davidge (2000). It can be checked in Davidge's Fig. 5 that it corresponds to an observed value of $H(\mathrm{HB})=13.5$ for NGC 6528, and the same value is found in Momany et al. (2003). For Terzan $5 H(\mathrm{HB})$ Cohn et al. adopt the dereddened value of $H(\mathrm{HB})=13.0$ whereas we have observed values of $H(\mathrm{HB})=13.76$ and 13.9 according to Stephens et al. or Cohn et al.'s calibrations. (ii) Cohn et al. used the Baade's Window Red Giant Branch as reference, and deduced $E(B-V)=2.18$ for Terzan 5. Using the distance from Ortolani et al. (2001) of $5.6 \mathrm{kpc}$, together with their lower reddening, a distance of $8.7 \mathrm{kpc}$ was found for Terzan 5 by Cohn et al.

In conclusion, the bright $H(\mathrm{HB})$ values in (i), and a low reddening of $(E(B-V)=2.18$ for Terzan 5 in (ii), may explain Cohn et al.'s different distance estimates relative to ours.
In the present work (Table 2), estimated distances of Terzan 5 are $6.3,4.6$ and $5.6 \mathrm{kpc}$. The three values are consistent, reinforcing the results, given that the first method is independent of photometric calibrations. An average distance $d_{\odot}=5.5 \pm 0.9 \mathrm{kpc}$ is adopted for Terzan 5.

Camilo \& Rasio (2005) presented evidence that globular clusters with pulsars have densities $\rho>1000 L_{\odot} / \mathrm{pc}^{3}$. In their Fig. 6 Terzan 5 is reported to be one of the densest clusters, with $\rho>10^{5.5} L_{\odot} / \mathrm{pc}^{3}$, assuming however a distance of $7.6 \mathrm{kpc}$ from the Sun (Harris 1996).

A closer distance causes a higher calculated stellar density $\left(L_{\odot} / \mathrm{pc}^{3}\right)$. Luminosity scales with $R^{2}$, whereas the volume scales with $R^{3}$, therefore the density increases for shorter distances. The present results imply that the density would increase by $30-40 \%$.

\section{Conclusions}

Using NICMOS on board the Hubble Space Telescope (Ortolani et al. 2001), we derive relative distances for Terzan 5, UKS 1 and Liller 1, based on the distance value of NGC 6528 derived by Momany et al. (2003). The distance of the metal-poor cluster Terzan 4 was derived from a comparison with M92 in the same NICMOS passbands.

From the diagram $J(\mathrm{HB})$ vs. $J-H(\mathrm{SGB} @ \mathrm{HB})($ Fig. 6), Terzan 5 is found to be closer to the Sun than NGC 6528 , whereas Liller 1 is at a comparable distance, and UKS 1 appears to be more distant. Assuming a distance to the Galactic center of $R_{\mathrm{GC}}=8 \mathrm{kpc}$ (Reid 1993), or closer distances of $R_{\mathrm{GC}}=7.2$ to $7.6 \mathrm{kpc}$ (Eisenhauer et al. 2005; Nishiyama et al. 2006; Bica et al. 2006), NGC 6528 is essentially at the Galactic center distance. Liller 1 could be somewhat beyond the Galactic center.

The relative metallicity of the sample metal-rich clusters, based on RGB slope in the infrared, show that NGC 6528 is the most metal-rich cluster, closely followed by Terzan 5 , Liller 1 and UKS 1 . Terzan 4 is clearly more metal-poor. The lower metallicity of UKS 1 favours the shorter distance of $9.3 \mathrm{kpc}$ based on Stephens et al. (2000)'s calibration, placing it in the bulge volume.

We have shown that Terzan 5 is at $d_{\odot}=5.5 \mathrm{kpc}$ from the Sun, thus significantly closer than the value of $d_{\odot}=8.7 \mathrm{kpc}$ calculated by Cohn et al. (2002), or $d_{\odot}=7.6 \mathrm{kpc}$ adopted by Camilo $\&$ Rasio (2005). Recently, Ransom (2006) pointed out that if the distance of Terzan 5 were really $8.7 \mathrm{kpc}$, then the current models of electron distribution in the Galaxy would overestimate the integrated electron density towards the cluster. For $8.7 \mathrm{kpc}$, models predict a dispersion measure of $530 \mathrm{pc} / \mathrm{cm}^{3}$, while for the pulsars in Terzan 5, the dispersion measured is estimated to be of $239 \mathrm{pc} / \mathrm{cm}^{3}$. This discrepancy is better explained by the distance of $5.5 \mathrm{kpc}$. The present shorter distance has also important implications concerning the cluster density. A 30-40\% decrease in distance using Camilo \& Rasio's (2005) value leads to a density increase by the same fraction, which is important for the calculation of the stellar collision rate, leading to the formation of low mass X-ray binaries and millisecond pulsars in this cluster (Edmonds et al. 2001; Ransom et al. 2005).

Acknowledgements. BB and EB acknowledge grants from CNPq and FAPESP. SO acknowledges the Italian Ministero dell'Università e della Ricerca Scientifica e Tecnologica (MURST) under the program on "Fasi iniziali di Evoluzione dell'Alone e del Bulge Galattico" (Italy). 


\section{Appendix A: JH calibrations of NICMOS photometry}

The calibrations we analysed in detail are:

a) Stephens et al. (2000):

$J=-0.344\left(m_{110}-m_{160}\right)+$ const.

$J-H=0.96\left(m_{110}-m_{160}\right)+$ const.

b) Cohn et al. (2002):

$J=-0.335\left(m_{110}-m_{160}\right)+$ const.

$J-H=0.68\left(m_{110}-m_{160}\right)+$ const.

c) Girardi et al. (2000) for $Z=0.02$ :

$J=-0.32\left(m_{110}-m_{160}\right)+$ const.

$J-H=0.77\left(m_{110}-m_{160}\right)+$ const.

d) Girardi et al. (2000) for $Z=0.0004$ :

$J=-0.2\left(m_{110}-m_{160}\right)+$ const.

$J-H=0.86\left(m_{110}-m_{160}\right)+$ const.

\section{A.1. Uncertainties in the colour transformation}

The errors on the distances and reddening values are dominated by the transformation coefficients (colour terms), from the NICMOS instrumental system, into the $J H$ magnitudes. More reddened clusters are more dependent on the reddening/calibration slopes, which is the case of UKS 1 and Liller 1.

The calibration of wide band photometry in non-standard systems, such as NICMOS, is not trivial. The estimation of uncertainties has no simple solution because standard stars are in general not available for extreme temperature and reddening values. Non-linearities and second order effects may be non negligible. In the following we analyse separately the two different calibrations that we used to derive the distances (Table 2), and compare them with other calibrations in the literature.

\section{A.2. Stephens et al.'s calibration}

Stephens et al.'s calibration (equations a) has a $J$ slope coefficient $(-0.344)$ similar to Cohn et al. (2002)'s calibrations, but their $H$ slope is larger $(-0.305$, vs. $\approx-0.1)$. This provides a flat slope for the $J-H$ transformation equation, and somewhat redder colours for very red stars. This explains the relatively shorter distance scale for the most reddened clusters (Liller 1, UKS 1), combined with high reddening.

Stephens et al. (2000) checked fits with simulations using infrared spectra of reddened stars. Assuming that bulge field giants are similar to cluster giants, this method potentially offers the most reliable transformations for bulge clusters. On the other hand, data for Baade's Window stars show some scatter, and they cover a limited colour range, producing errors of \pm 0.1 on the colour slope and \pm 0.08 mag. on the zero point, and an error up to $\pm 0.2 \mathrm{mag}$ at the extremes of the colours of the sample clusters.

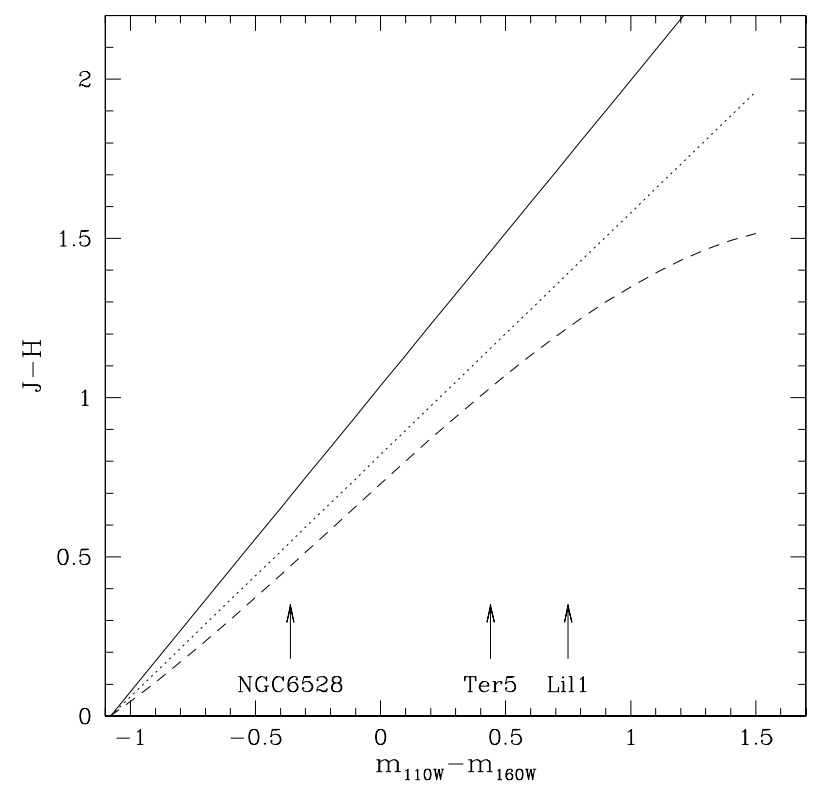

Fig. A.1. Colour calibrations from Stephens et al. (2000) (solid line), Cohn et al. (2002) (dotted) and Origlia \& Leitherer (2000) (dashed). The $m_{110}-m_{160}$ colours of NGC 6528, Terzan 5 and Liller 1 are shown.

\section{A.3. Cohn et al.'s calibration}

The calibration used by Cohn et al. (2002) (equations b) is based on the NICMOS five solar neighbourhood standard stars, distributed over a wide temperature/colour range. The $J-H$ colour term is lower than in Stephens et al.'s calibration (0.76 vs. 0.96), resulting in bluer colours and increasing distances for more reddened clusters (Table 2). The formal errors, from the linear interpolation, are similar to those in Stephens et al. They are marginally compatible with the high metallicity of Liller 1 and UKS 1, and with Liller $1 J H K$ photometry (Frogel et al. 1995). The JH CMDs of NGC 6528 calibrated with Stephens et al. and Cohn et al.'s equations have been compared with Momany et al. (2003)'s ground based photometry. The colours were found in better agreement with Stephens et al.'s calibration, whereas Cohn et al.'s calibration gave somewhat bluer colours.

\section{A.4. Other calibrations}

Cohn et al.'s calibration equations are similar to others in the literature, such as Macri et al.'s (2001). In the latter case the calibration was derived from solar metallicity Kurucz models, convolved with NICMOS passbands and compared to the standard $J H$ system. Reddening effects were not considered. They derived small formal errors, one order of magnitude less than those of Cohn et al. (2002).

A colour calibration was obtained by Origlia \& Leitherer (2000). They used Bessell et al. (1988) model atmospheres to transform NICMOS colours into the JHK system, and concluded that the transformations are gravity and metallicity independent, but a cubic polynomial relation is required to fit the data. The effect of such an interpolation is that for NGC 6528 and Terzan 5, with reddening values lower than UKS 1 and Liller 1, the results are similar to Cohn et al. (2002) and Macri et al. (2001) calibrations, but the colours are considerably bluer for more reddened clusters, implying very large distances for UKS 1 and Liller 1. The effect of linearity deviations is seen in their Fig. 11, as well as a discrepancy with the reddest standard stars. Figure A.1 
shows the different colour calibrations in the metallicity range of our clusters.

From the analyses above we conclude that systematic errors of the order of $\pm 0.2-0.3 \mathrm{mag}$., related mostly to colour transformation slopes, affect distance moduli, in particular those of the most reddened clusters.

\section{A.5. Calibrations with Padova stellar evolution models}

In order to clarify the nature of the discrepancies between the described calibrations, we performed a further independent test based on the Girardi et al. (2002) NICMOS isochrones in the $m_{110}$ and $m_{160}$ bands. The authors derived the isochrones from ATLAS9 models (Castelli et al. 1997) and a NICMOS transmission system provided by the NICMOS staff. Comparing these isochrones with those by Girardi et al. (2000) in the JHK system, we obtained the transformation equations c) and d) for metallicities of $Z=0.019$ (solar), and a low metallicity of $Z=0.0004$, in the full colour range of the isochrones (about $0.2<m_{110}-m_{160}<$ 1.1). For solar metallicity the linear interpolation gives a colour term of -0.32 , somewhat lower than that obtained by Stephens et al. (2000). At lower metallicities the coefficient decreases to -0.2 at $Z=0.0004$. The colour coefficients in this calibration are intermediate between Stephens et al. and Cohn et al.'s. The result of this test indicates that the metallicity is not the major source of the discrepancy between Stephens et al. (2000) and the synthetic photometry. We argue that an important source of discrepancy could be reddening, in agreement with Lee et al. (2001). Using their reddening law calculated for NICMOS bands they derived an average $A\left(m_{110}\right) / E(B-V)=1.16$ (for SGB and RGB stars), while Rieke \& Lebofsky (1985) give $A_{J} / E(B-V)=0.864$. A star reddened by one magnitude in the $m_{110}-m_{160}$ colour will imply $A\left(m_{110}\right)-A_{J}=0.55 \mathrm{mag}$. It is evident that the colour terms coming from synthetic photometry are by far too small to account for the reddening effects.

We conclude that a correct calibration cannot be obtained if the reddening is not known in advance. A solution could be an iterative process deriving a first guess reddening and proceeding further with subtraction of the reddening and calibration of the dereddened data, adding the obtained final reddening to the calibrated data.

\section{References}

Barbuy, B., Bica, E., \& Ortolani, S. 1998, A\&A, 333, 117

Bica, E. 1988, A\&A, 195, 76

Bica, E., Bonatto, C., Barbuy, B., \& Ortolani, S. 2006, A\&A, 450, 105

Cackett, E. M., Wijnands, R., Heinke, C. O., et al. 2006, MNRAS, 369, 407

Camilo, F., \& Rasio, F. A. 2005, ASP Conf. Ser., 328, 147

Carretta, E., Cohen, J. G., Gratton, R. G., \& Behr, B. B. 2001, AJ, 122, 1469

Castelli, F., Gratton, R. G., \& Kurucz, R. L. 1997, A\&A, 318, 841

Cohn, H. N., Lugger, P. M., Grindlay, J. E., \& Edmonds, P. D. 2002, ApJ, 571, 818

Davidge, T. J. 2000, ApJS, 126, 105

Edmonds, F. D., Grindlay, J. E., Cohn, H., \& Lugger, P. 2001, ApJ, 547, 829

Eisenhauer, F., Genzel, R., Alexander, T., et al. 2005, ApJ, 628, 246

Fitzpatrick, I. L. 1999, PASP, 111, 63

Frogel, J. A., Kuchinski, L. E., \& Tiede, G. P. 1995, AJ, 109, 1154

Girardi, L., Bressan, A., Bertelli, G., \& Chiosi, C. 2000, A\&AS, 141, 371

Girardi, L., Bertelli, G., Bressan, A., et al. 2002, A\&A, 391, 195

Harris, W. E. 1996, AJ 112, 1487

Heinke, C. O., Wijnands, R., \& Cohn, H. N. 2006, ApJ, 651, 1098

Hut, P., McMillan, S., Goodman, J., et al. 1992, PASP, 104, 981

Kim, S. S., Figer, D. F., Lee, M. G., \& Oh, S. 2005, PASP, 117, 445

Lee, J.-W., Carney, B. W., Fullton, L. K., \& Stetson, P. B. 2001, AJ, 122, 3136

Lorimer, D. R. 2005, Living Reviews in Relativity, 8, 7

Macri, L. M., Calzetti, D., Freedman, W. L., et al. 2001, ApJ, 549, 721

Matsunaga, N., Deguchi, S., Yoshifusa, I., et al. 2005, PASJ, 57, L1

Momany, Y., Ortolani, S., Held, E. V., et al. 2003, A\&A, 402, 607

Nishiyama, S., Nagata, T., Sato, S., et al. 2006, ApJ, 647, 1093

Origlia, L., \& Leitherer, C. 2000, AJ, 119, 2018

Origlia, L., \& Rich, R. M. 2004, AJ, 127, 3422

Origlia, L., Rich, R. M., \& Castro, S. 2002, AJ, 123, 1559

Origlia, L., Valenti, E., Rich, R. M., \& Ferraro, F. R. 2005, MNRAS, 363, 897

Ortolani, S., Barbuy, B., \& Bica, E. 1990, A\&A, 236, 362

Ortolani, S., Barbuy, B., \& Bica, E. 1991, A\&A, 249, L31

Ortolani, S., Bica, E., \& Barbuy, B. 1992, A\&AS, 92, 441

Ortolani, S., Renzini, A., Gilmozzi, R., et al. 1995, Nature, 377, 701

Ortolani, S., Barbuy, B., \& Bica, E. 1996a, A\&A, 308, 733

Ortolani, S., Bica, E., \& Barbuy, B. 1996b, A\&A, 306, 134

Ortolani, S., Barbuy, B., \& Bica, E. 1997a, A\&A, 319, 850

Ortolani, S., Bica, E., \& Barbuy, B. 1997b, A\&AS 126, 319

Ortolani, S., Barbuy, B., Bica, E., Renzini, A., \& Zoccali, M. 2001, A\&A, 376, 878

Ransom, S. M., Hessels, J. W. T., Stairs, I. H., et al. 2005, Science, 307, 892

Ransom, S. M. 2006, Small Ionized and Neutral Structures in the Diffuse Interstellar Medium, ASP Conf., ed. M. Haverkorn, \& W.M. Goss, in press [arXiv: astro-ph/0611672]

Reid, M. J. 1993, ARA\&A, 31, 345

Schlegel, D. J., Finkbeiner, D. P., \& Davis, M. 1998, ApJ, 500, 525

Stephens, A. W., Frogel, J. A., Ortolani, S., et al. 2000, AJ, 119, 419

Tiede, G. P., Martini, P., \& Frogel, J. A. 1997, AJ, 114, 694

Verbunt, F., \& Hut, P. 1987, in The Origin and Evolution of Neutron Stars, ed. D. J. Helfand, \& J. Huang (Dordrecht: Reidel), 187

Zoccali, M., Barbuy, B., Hill, V., et al. 2004, A\&A, 423, 507 